\title{
What to look forward to in GaBI Journal, 2016, Issue 2
}

\section{Professor Philip D Walson, MD}

This second issue of 2016 includes manuscripts that cover a number of important issues of interest to GaBI Journal including methods used to assess bioequivalence and decide on interchangeability, patient and advocacy group opinions concerning the use of follow-on products, batch-to-batch consistency in monoclonal antibody production, and the causes and potential ways to deal with the mismatch between potential savings and actual use of generics and biosimilars. The comments, opinions and data presented demonstrate the general lack of consensus about how generics and biosimilars should be regulated, used and monitored as well as the different approaches being taken in an attempt to deal with some of the causes of this lack of consensus.

The first two papers discuss the use of adjusted indirect comparisons between generics, which are used by regulators to decide on bioequivalence and interchangeability of generic drug products. In the Editorial Professors Laszlo Endrenyi and László Tóthfalusi discuss the conclusions presented in the Review Article by Gwaza et al. who concluded that, 'adjusted indirect comparison is a useful tool to compare relative bioavailabilities between generics'. While agreeing in general with this conclusion, Professors Endrenyi and Tóthfalusi warn that there are limitations to such comparisons and that to use this method reliably, the ratios of the point estimates between the generics should be small and the original studies should be sufficiently powered'.

When reading the manuscripts in this issue I was reminded of how difficult it was to teach pharmacokinetics and therapeutic drug monitoring to those (many) clinicians who were both uncomfortable with and usually poorly trained in mathematics. I personally suspect that the reasons that regulators and pharmacists are generally more willing to accept generics and biosimilars is that they are more likely to understand the scientific rationale upon which their approval is based. Other manuscripts, especially those very skeptical of regulatory approval decisions also reminded me that we as humans are skeptical of and resistant to things we do not understand. I am influenced no doubt in this belief by what Mr Alvin Toffler, the author of Future Shock who died on 27 June 2016 at age 87, wrote over 45 years ago. Writing during the late 1960s Toffler summarized this thesis thus: in three short decades between now and the turn of the next millennium, millions of psychologically normal people will experience an abrupt collision with the future. Affluent, educated citizens of the world's richest and most technically advanced nations, they will fall victim to tomorrow's most menacing malady: the disease of change. Unable to keep up with the supercharged pace of change, brought to the edge of breakdown by incessant demands to adapt to novelty, many will plunge into future shock. For them, the future will have arrived too soon. [Toffler A. Quoted. In: Cross N, editors. Man made futures. London: Hutchinson; 1974].

The third paper by Mr Brian J Malkin is described well by its title, Biosimilars patent litigation in Canada and Japan: a comparative strategic overview and EU and US update. The author is the Senior Counsel for a legal firm that deals with biosimilar patent law and reviews important legal strategies being used. While perhaps most appealing to pharmaceutical and payer legal departments, it also is of interest I feel for the journal's more general readership which is why we have included it in a new, 'Legal' section of the journal.

Many entities, including the GaBIJournal, have begun efforts to 'educate' all stakeholders in the hope that this will improve understanding and therefore promote the most appropriate regulation, use and monitoring of follow-on products and allow for these products to provide the cost savings that they could offer. This approach is evidenced by the first Perspective paper by Azevedo et al. entitled, New monoclonal antibody biosimilars approved in 2015 in Latin America: position statement of the Latin American Forum on

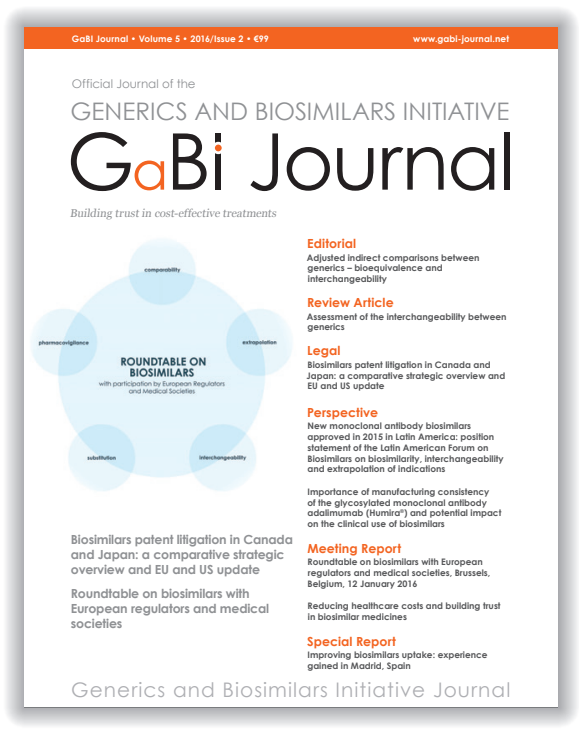

Biosimilars on biosimilarity, interchangeability and extrapolation of indications which concluded that, "Extrapolation is only acceptable when the diseases for which the reference product is intended to treat are entirely similar. Extrapolation based on only preclinical studies is not acceptable. Conversely, although the proposed rituximab biosimilar (RTXM83) was approved by ANMAT (National Administration for Medicines, Food and Medical Technology) in Argentina, clinical data demonstrating its equivalence with the reference rituximab, is necessary before RTXM83 can be considered a true biosimilar'.

It was actually difficult to decide under which editorial section of the journal the Azevedo et al. perspective paper should be placed. It could have been a meeting report for the recent (29-30 June 2016) Latin American Forum on Biosimilars (FLAB) conference held in Brasilia, Brazil but the authors state that, 'We have established a FLAB position statement on the approval of these drugs in the context of the current regulations in Brazil and Argentina'. Unfortunately, it is not clear neither who 'we' represents other than the five authors nor how this statement/paper was developed nor which of the speakers, organizers, sponsors or participants actually approved this 'position statement'. Readers are directed to the FLAB website to review the organizers, speakers and topics presented at this conference but it should be noted that the FLAB organizers/ authors are on Advisory Boards and speakers panels for pharmaceutical firms which raises some questions about potential bias. 
The lack of clarity in this paper contrasts with the Meeting Report by Annese et al. that describes the Roundtable on biosimilars with European regulators and medical societies held in Brussels, Belgium on 12 January 2016. This report reviewed essentially the same data and similar topics, but it much more clearly presents the methods used to discuss the manufacturing and characterization of biologicals, clinical and non-clinical comparability, immunogenicity, extrapolation, interchangeability, and pharmacovigilance of biosimilars in order to come to the conclusions reached that, 'The picture of biosimilars is becoming clearer, and stakeholders are beginning to understand better the basis of biosimilar development, on one hand, and the reasons for concerns, on the other hand. Different stakeholders - patients, doctors, pharmacists, payers - need different information. Above all, this must be a collaborative exercise'. The authors of this meeting report represent three different stakeholders: physicians, academia and regulatory authority.

The second Perspective paper by Declerck et al. entitled, Importance of manufacturing consistency of the glycosylated monoclonal antibody adalimumab (Humira®) and potential impact on the clinical use of biosimilars reviews the previously reported batch-to-batch performance consistency for an important anti-TNF $\alpha$ monoclonal antibody product [see Tebbey PW, Varga A, Naill M, Clewell J, Venema J. Consistency of quality attributes for the glycosylated monoclonal antibody Humira ${ }^{\circledR}$ (adalimumab). MAbs. 2015;7(5):805-11]. The history of this product and the manufacturing data reviewed both have important implications for a number of reasons. The product was approved initially (in 2002) in the US only for treatment of rheumatoid arthritis. Later it was approved for the management of Crohn's disease, ulcerative colitis, plaque psoriasis, psoriatic arthritis, ankylosing spondylitis, juvenile idiopathic arthritis, and hidradenitis suppurativa. This history is important for the 'mechanism-based' approach to extrapolation of indications. The fact that, 'It has been clearly demonstrated that the manufacturing process for adalimumab produced a consistent product over an extended ( $>10$ years) period of time, even as necessary changes to the manufacturing processes and production scale were introduced', is important both because it shows that this is possible and because it establishes the range of differences in 'key physicochemical and functional quality attributes' that can be expected when a patient is continued on this 'same' name product over time. Such data can then be used to 'set the bar' for how much different a new (biosimilar) product can be allowed to be without expecting to produce clinically meaningful differences in clinical responses. Additionally, the preclinical and immunological data monitoring methods used and results produced can be used to assess follow-on product performance. Finally, such data are important to present to clinicians and patients so they can understand how much product variability they will be exposed to over time even if they continue to receive only the same, named product so that this variability can be compared to that present in a biosimilar product.

Despite growing evidence that it is possible to produce and use reliable generic and biosimilar products, their uptake remains a problem as evidenced by a
Special Report entitled, Improving biosimilars uptake: experience gained in Madrid, Spain by Dr Michelle Derbyshire, our GaBI Online Editor, who summarizes a part of the paper by Oyarzábal et al. from the Madrid Health Service. These investigators reported tremendous variability (from $0 \%$ to $70 \%$ ) in the uptake of approved somatropin, erythropoietin or filgrastim biosimilars by a number of Madrid hospitals. And while the average uptake increased slightly (from 15.72\% in 2014 to $20.07 \%$ in 2015), despite the methods being used to encourage it, the uptake was still far below both the highest and potential use possible.

At least part of the reasons for the disconnect between the potential and actual healthcare cost savings provided by the use of generics and biosimilars is to be found in a review of two recent studies (PLANETAS and PLANETRA) discussed at the two meetings mentioned above (the FLAB meeting in Brasilia and the GaBI roundtable in Brussels). Results of these two extension studies are presented in abstracted form in two Pharma News papers, which summarize the results of switching from the infliximab reference product to CT-P13 in patients with rheumatoid arthritis or ankylosing spondylitis.

As always, whether impacted by Future Shock or not, our readers are encouraged to submit their comments or concerns as well as their own manuscripts.

Professor Philip D Walson, MD Editor-in-Chief, GaBiJournal

DOI: 10.5639/gabij.2016.0502.013

Copyright (c) 2016 Pro Pharma Communications International 